\title{
Relación entre nivel de conocimiento sobre factores de riesgo cardiovascular y conducta de riesgo cardiovascular referida por las enfermeras
}

\author{
Ysabel Sandoval-Morillo ${ }^{1, a}$, Hilda Lastrera- Obregón a ${ }^{\text {a }}$, Silvia Espinoza-Saraviaa ${ }^{2, a}$, Clara Torres-Deza ${ }^{3, b, c}$ \\ RESUMEN
}

Objetivos: Determinar la relación entre el nivel de conocimiento sobre los factores de riesgo cardiovascular modificables y la conducta de riesgo cardiovascular referida por las enfermeras del Hospital Nacional Arzobispo Loayza. Material y método:el estudio fue descriptivo, correlacional. La muestra estuvo conformada por 107 enfermeras del Hospital Arzobispo Loayza. El muestreo fue probabilístico aleatorio simple, la recolección de datos se hizo a través de la técnica de encuesta, cuyo instrumento fue un cuestionario elaborado por las investigadoras y pasó por un proceso de validación con la correlación de Pearson, y la prueba alfa de Cronbach, para su confiabilidad. Resultados: El 56\% del personal de enfermería posee un nivel de conocimiento medio acerca de los factores de riesgo cardiovascular modificable, y el 45\% tiene una conducta de alto riesgo. Conclusiones:no existe relación entre el nivel de conocimientos acerca de los factores de riesgo cardiovascular y la conducta de riesgo cardiovascular en enfermeras.

PALABRAS CLAVE: Conocimiento, conducta de riesgo, factor de riesgo cardiovascular modificable.

\section{Relationship between level of knowledge about cardiovascular risk factors and cardiovascular risk behavior referred by nurses}

\section{SUMMARY}

Objectives:To determine the relationship between the level of knowledge about modifiable cardiovascular risk factors and cardiovascular risk behavior referred by nurses Hospital Nacional Arzobispo Loayza. Material and Methods: The study was descriptive correlational. The sample was 107 Hospital Nacional Arzobispo Loayza nurses, selected according to the inclusion criteria of a total of 423 nurses. The simple random sampling was probabilistic, data collection was done through the survey technique, the instrument was a questionnaire developed by the researchers themselves and went through a validation process with Pearson correlation and Cronbach's alpha test for its reliability. Results: $56 \%$ of the nurses has a medium level of knowledge about modifiable cardiovascular risk factors and $45 \%$ had high-risk behavior. Conclusion: There is no relationship between the level of knowledge about cardiovascular risk factors and cardiovascular risk behavior in nurses.

KEY WORDS: Knowledge, risk behavior, modifiable cardiovascular risk factor.

${ }^{1}$ Laboratorio de Análisis, Clínico SISALAB.Lima, Perú.

${ }^{2}$ Clínica San Felipe. Lima, Perú

${ }^{3}$ Facultad de Enfermería, Universidad Peruana Cayetano Heredia. Lima, Perú.

${ }^{a}$ Licenciada en Enfermería ; ${ }^{b}$ Docente Asociada ; ${ }^{\circ}$ Magister en Salud Pública. 


\section{INTRODUCCIÓN}

Las enfermedades cardiovasculares (ECV) tienen enorme relevancia a nivel mundial debido a las elevadas tasas de morbimortalidad, el grado de discapacidad que producen y las repercusiones sociales y económicas (1). Cada año mueren más personas por ECV que por cualquier otra causa; afectan por igual a ambos sexos, y más del $80 \%$ se producen en países de ingresos bajos y medios. Se calcula que las muertes por enfermedad cardiovascular aumentarán de 17 millones en el 2008 a 25 millones en el 2030 (1,2). La Sociedad Peruana de Cardiología, al estudiar el Registro Nacional de Infarto miocárdico agudo (RENIMA - 2008) encontró que alrededor de tres infartos se registraban diariamente en la población adulta, y que la mortalidad era del $7,4 \%$ sin diferencias significativas tanto en Lima como en provincias (3).

Según el Framingham heart study, desde la década de los 40 se realizaron estudios con el propósito de identificar los factores de riesgo relacionados con enfermedades cardiovasculares. Se encontró que había asociación de la enfermedad coronaria con el tabaquismo, la obesidad, la actividad física, la dieta y el consumo de alcohol (4). Estas conductas de riesgo, dan lugar al aumento de la tensión arterial, la glucemia, las concentraciones anormales de lípidos en sangre, el sobrepeso y la obesidad (5). Estos comportamientos han sido construidos socialmente, y no pueden ser modificados necesariamente por el conocimiento, debido a que existen factores condicionantes, tanto biológicos como hábitos adquiridos, que predicen el comienzo de la enfermedad cardiovascular. La alta prevalencia de estas enfermedades y las medidas individuales para reducirlas, hacen pensar que las personas requieren algo más que conocimientos para cambiar sus estilos de vida (6).

Así también, el consumo de drogas legales como el alcohol y el tabaco, ejercen mayor impacto sobre la salud cardiovascular (7-10). Por consumo de alcohol mueren 2,3 millones de personas al año a nivel mundial, y es el primer factor de riesgo en el pacífico occidental y las Américas (11). En el Perú, el abuso y la dependencia al alcohol es la tercera causa de pérdidas económicas por muerte o discapacidad (12). Es una droga socialmente aceptada y de mayor consumo en todas las edades, en ambos sexos y en todos los grupos sociales (13); uno de sus componentes es el etanol, el cual es un agente tóxico que tiene efectos agudos y crónicos sobre el sistema cardiovascular y aumenta el riesgo de sufrir enfermedades cerebrovasculares isquémicos y hemorrágicos. Produce insuficiencia cardiaca, arritmias y depresión de la contractilidad miocárdica, por la liberación de catecolaminas desde la médula suprarrenal (14).

Por otro lado, por adicción al tabaco muere una persona cada 6 segundos. Uno de cada dos fumadores morirá por adicción al tabaco, específicamente por la nicotina, que es la que causa adicción (15). En el Perú, se estima 9000 defunciones al año a consecuencia del consumo de tabaco (16). Las mujeres que fuman tienen un mayor riesgo de enfermedad cardiovascular incluyendo enfermedad coronaria, ictus isquémico, hemorragia subaracnoidea, infarto de miocardio y muerte súbita (17).

Las personas que consumen cigarrillos tienen una probabilidad de 2 a 3 veces más riesgo de desarrollar enfermedad coronaria que los que no fuman y, además, tienen una probabilidad cinco veces más alta morir por causa de infarto agudo de miocardio. Asimismo, aumenta el riesgo de desarrollo de aneurisma aórtico, enfermedad vascular periférica y evento cerebrovascular isquémico (18). Las personas que dejan de fumar reducen el riesgo de un evento coronario en un $50 \%$ en los primeros 1 a 2 años; después de suspender el hábito el riesgo se aproxima al de los no fumadores después de 5 a 15 años $(17,18)$.

Otro aspecto por considerar está relacionado con la falta de actividad física, el cual es un factor de riesgo potencialmente modificable, y que debería recibir mayor atención para reducir el impacto de la enfermedad coronaria cardiaca en la sociedad $(19,20)$. Según la OMS, la inactividad física causa la muerte de 2 millones de personas en el mundo al año. Además, el $60 \%$ de la población, no llega a realizar los 30 minutos diarios de ejercicio físico que recomienda la OMS (19). La tendencia hacia un estilo de vida inactiva y sedentaria es más frecuente en el grupo de edad adulta, especialmente dentro de la población femenina (21); por ser un momento de la vida en que asume numerosas responsabilidades laborales y familiares (22). La recomendación de realizar ejercicio físico, ha pasado a ser un elemento importante de las políticas preventivas en los adultos, los ancianos y los niños (23).

En consideración a lo expuesto, los profesionales de enfermería no solo deben inculcar hábitos de vida saludable, sino también internalizar la información y apropiarse de ella, para conseguir estilos de vida saludables con conductas positivas en cuanto a una dieta equilibrada, ejercicio físico en forma regular y abstención del consumo de alcohol y cigarrillos. Cuidar de sí mismo es esencial, tanto para sentirse bien en el ambiente de trabajo como 
para cuidar mejor de otros (24). Asimismo, para evitar la discrepancia entre lo que se dice y se hace, ya que las enfermeras están llamadas a promocionar conductas favorecedoras para prevenir la enfermedad, más aun cuando se trata de factores de riesgo modificables.

Dorotea Orem, enfermera teórica refiere que el autocuidado es una actividad aprendida y que debe aplicarse de forma deliberada y continua a lo largo del tiempo. Uno de los tipos de autocuidado para Dorothea Orem son los derivados de las necesidades básicas, en sus etapas de crecimiento y desarrollo, estados de salud, características sanitarias y factores del entorno que posee cada individuo (25). Estas necesidades han sido estructuradas por la misma cultura, por lo que siempre estarán implícitas en el estilo de vida de todas las personas. Por lo tanto, la persona que es autosuficiente es responsable de sus propios cuidados y de las personas que tiene a su cargo; debido a que tendrá capacidades para dar respuesta a toda una serie de necesidades para la vida. Ello debe suponer la necesidad de llevar una vida sana, comportarse de tal forma que pueda alcanzar el bienestar y la salud.

El objetivo del estudio fue determinar la relación entre el nivel de conocimiento acerca de los factores de riesgo cardiovascular y conducta de riesgo cardiovascular modificable referido por las enfermeras del Hospital Nacional Arzobispo Loayza.

\section{MATERIAL Y MÉTODOS}

Estudio descriptivo de corte transversal, con diseño correlacional. Se realizó en los servicios de hospitalización de medicina, cirugía, especialidades y consultorios externos del Hospital Nacional Arzobispo Loayza.

La población estuvo constituida por 423 profesionales de enfermería del Hospital Nacional Arzobispo Loayza, se tuvieron en cuenta los siguientes criterios de selección: enfermeras(os) que tengan relación laboral con el hospital Loayza, que aceptenparticipar en la investigación y que no presenten enfermedad cardiovascular. No se incluyó a las enfermeras especialistas en cardiología.

La muestra estuvo constituida por 107 enfermeras de los servicios de hospitalización. El tamaño muestral se determinó mediante la prueba del coeficiente de correlación de Pearson

Para seleccionar la muestra de cada área de servicio se aplicó la fijación proporcional y la selección de enfermeras fue aleatoria. Según área de servicio la muestra fue de la siguiente manera: hospitalización medicina 26; de cirugía 11; de los servicios de especialidades 23 ; de consultorios externos 10; de las unidades críticas 18; de sala de operaciones 1 , y del área administrativa 4 . Se aplicó una encuesta y un cuestionario elaborado por las investigadoras, que se divide en 3 secciones: la primera, datos generales (edad, sexo, lugar de residencia, estado civil, número de hijos, área donde labora, tiempo de servicio, contorno de cintura, peso, talla y presión arterial).La segunda parte presenta 18 preguntas sobre riesgo cardiovascular, con cuatro alternativas y una respuesta correcta. La tercera parte mide las conductas de riesgo cardiovascular con 10 preguntas, con cuatro alternativas y una respuesta correcta.

La validez del instrumento, se realizó mediante juicio de expertos, conformado por un equipo multidisciplinario: las opiniones de los jueces fueron evaluadas mediante la prueba AIKEN. La validación del instrumento se realizó mediante el coeficiente de correlación de Pearson en una prueba piloto con 25 enfermeras. Se evaluó la confiabilidad mediante la prueba alfa de Cronbachel procesamiento de datos se realizó mediante el software estadístico SPSS 18 , obteniendo cuadros y gráficos.

Con respecto al análisis e interpretación de los resultados, en la primera fase, se calculó la suma de los puntajes de los 18 ítems, el cual varía de 0 a 18 puntos (se dio un punto por cada respuesta correcta y cero puntos para cada incorrecta), y se clasificó según escala de Staninos, en el nivel conocimiento: alto, medio y bajo. Para conducta de riesgo se calculó la suma de los puntajes de los 17 ítems, el cual varía de 0 a 52 puntos (se dio 4 puntos a la que represente mayor conducta de riesgo y cero puntos a la que

Tabla 1. Nivel de conocimiento acerca de los factores de riesgo cardiovascular modificable en las enfermeras del Hospital Nacional Arzobispo Loayza, diciembre 2012.

\begin{tabular}{ccc}
\hline Conocimiento & Número & Porcentaje \\
\hline Alto & 15 & 14 \\
Medio & 60 & 56 \\
Bajo & 32 & 30 \\
Total & 107 & 100 \\
\hline
\end{tabular}


Tabla 2. Nivel de conocimiento acerca de los factores de riesgo cardiovascular modificable que tienen las enfermeras según dimensiones.Hospital Nacional Arzobispo Loayza,diciembre 2012.

\begin{tabular}{ccccccccc}
\hline DIMENSIONES & \multicolumn{2}{c}{ Alto } & \multicolumn{2}{c}{ Medio } & \multicolumn{2}{c}{ Bajo } & \multicolumn{2}{c}{ Total } \\
& N. ${ }^{\circ}$ & $\%$ & N. ${ }^{\circ}$ & $\%$ & N. ${ }^{\circ}$ & $\%$ & N.. & $\%$ \\
\hline Conocimiento sobre drogas legales & 14 & 13 & 59 & 55 & 34 & 32 & 107 & 100 \\
$\begin{array}{c}\text { (alcohol y tabaco) } \\
\text { Conocimiento sobre ejercicio físico }\end{array}$ & 22 & 21 & 52 & 49 & 33 & 30 & 107 & 100 \\
Conocimiento sobre hábitos alimenticios & 8 & 8 & 71 & 66 & 28 & 26 & 107 & 100 \\
\hline
\end{tabular}

Tabla 3. Conducta de riesgo cardiovascular modificable referidapor las enfermeras. Hospital Nacional Arzobispo Loayza,diciembre 2012.

\begin{tabular}{ccc}
\hline Conducta de riesgo & Número & Porcentaje \\
\hline Conducta de alto riesgo & 48 & 45 \\
Conducta de mediano riesgo & 9 & 8 \\
Conducta de bajo riesgo & 50 & 47 \\
Total & 107 & 100 \\
\hline
\end{tabular}

Tabla 4. Conducta de riesgo cardiovascular modificable referidapor las enfermeras según dimensiones.

Hospital Nacional Arzobispo Loayza,diciembre 2012.

\begin{tabular}{cccccccccc}
\hline Dimensiones: & \multicolumn{2}{c}{ Alto riesgo } & \multicolumn{2}{c}{ Mediano riesgo } & Bajo riesgo & \multicolumn{2}{c}{ Total } \\
\hline conducta de riesgo & $\mathrm{N} .{ }^{\circ} . \cdot$ & $\%$ & $\mathrm{~N} .{ }^{\circ} \cdot$ & $\%$ & $\mathrm{~N} .{ }^{\circ}$. & $\%$ & $\mathrm{~N} .{ }^{\circ}$. & $\%$ \\
Droga legal alcohol & 3 & 3 & 15 & 14 & 89 & 83 & 107 & 100 \\
Droga legal tabaco & 5 & 5 & 2 & 2 & 100 & 93 & 107 & 100 \\
Ejercicio físico & 93 & 87 & 9 & 8 & 5 & 5 & 107 & 100 \\
Hábitos alimenticios & 92 & 86 & 10 & 9 & 5 & 5 & 107 & 100 \\
\hline
\end{tabular}

Tabla 5. Relación entre nivel de conocimiento sobre factores de riesgo cardiovascular y conducta de riesgo cardiovascular modificable referida por las enfermeras. Hospital Nacional Arzobispo Loayza, diciembre 2012.

\begin{tabular}{cccccccccc}
\hline Conocimiento & \multicolumn{3}{c}{ Conducta de riesgo } & \multicolumn{3}{c}{ Total } \\
& \multicolumn{2}{c}{ Alto riesgo } & Mediano riesgo & Bajo riesgo & \\
\hline & $\mathrm{N} .^{\circ}$ & $\%$ & $\mathrm{~N} .{ }^{\circ}$ & $\%$ & $\mathrm{~N} .{ }^{\circ}$ & $\%$ & $\mathrm{~N}^{\circ}{ }^{\circ}$ & $\%$ \\
Alto & 5 & 5 & 2 & 2 & 8 & 7 & 15 & 14 \\
Medio & 26 & 24 & 6 & 6 & 28 & 26 & 60 & 56 \\
Bajo & 17 & 16 & 1 & 1 & 14 & 13 & 32 & 30 \\
Total & 48 & 45 & 9 & 8 & 50 & 47 & 107 & 100 \\
\hline
\end{tabular}

$\mathrm{p}>0,05$ no significativa 
represente menor conducta de riesgo), y se clasificó según escala de Staninos, en conducta de alto riego, mediano y bajo riesgo. En la segunda fase, se estableció la relación entre las variables mediante la aplicación de la prueba estadística dechi-cuadrado.

\section{RESULTADOS}

En contramos que un 56\% tienen un nivel de conocimiento medio acerca de los factores de riesgo cardiovascular modificable, un 30\% tiene conocimiento bajo (Tabla 1).

El nivel de conocimiento es medio en las tres dimensiones: Conocimiento sobre drogas legales (alcohol y tabaco) un $55 \%$, conocimiento sobre ejercicio físico un $49 \%$ y Conocimiento sobre hábitos alimenticios un 66\% (Tabla 2).

Del total de profesionales de enfermería un $47 \%$ tienen una conducta de bajo riesgo cardiovascular modificable, un $45 \%$ tiene una conducta de alto riesgo y finalmente, un $8 \%$ tiene una conducta de mediano riesgo (Tabla 3 ).

En el ejercicio físico hay conducta de alto riesgo en un $87 \%$ y en hábitos alimentarios un $86 \%$ de conducta de riesgo (Tabla 4$)$.

En la tabla 5 se aprecia que no existe relación significativa entre nivel de conocimiento y conducta de riesgo sobre factores de riesgo cardiovascular en enfermeras, que las enfermeras que presentan un nivel de conocimiento alto muestran una conducta de riesgo bajo en un 7\%, y un 5\% de conducta de alto riesgo y que las enfermeras que tienen un nivel de conocimiento medio tienen una conducta de riesgo bajo en un $26 \%$, seguido de un $24 \%$ con una conducta de alto riesgo.Los que tienen nivel de conocimiento bajo muestran una conducta de riesgo alto en un $16 \%$, y un $13 \%$ presentan conducta de riesgo bajo.

Por tanto, no existe relación significativa entre conocimiento y conducta de riesgo cardiovascular referido por las enfermeras, es decir; a conocimiento alto es poco probable que tenga conducta deriesgo bajo y a bajo conocimiento es poco probable que haya conducta de riesgo alto; según prueba estadística chi cuadrado $(\mathrm{p}>0,05)$

Además, aplicando el coeficiente de correlación de Spearman no existe correlación entre el nivel de conocimiento y conducta de riesgo cardiovascular modificable referida por las enfermeras.

\section{DISCUSIÓN}

En el estudio se encontró que el nivel de conocimientos acerca de los factores de riesgo cardiovascular es medio $(56 \%)$ seguido de conocimiento bajo (30\%) (Tabla 1). Sobre el conocimiento sobre drogas legales (alcohol y tabaco), se encontró que un 55\% tiene un nivel de conocimiento medio con una tendencia a conocimiento bajo (Tabla 2). Esto difiere de lo encontrado por López E. en su estudio conocimiento de los factores de riesgo cardiovascular y comportamiento para la prevención del personal de enfermería quienes tienen un conocimiento acertado en un $85,7 \%$ (26).

El conocimiento medio y bajo de las enfermeras podría deberse a que las instituciones formadoras de recursos humanos en salud consideran en su currículoeducativo la promoción de la salud, pero no cuentan con una formación específica respecto al consumo de drogas legales que les permita obtener mayores conocimientos y que sus actitudes les permitan enfrentar los problemas del consumo de estas drogas durante el ejercicio profesional futuro. Asimismo, los programas de educación continua en las instituciones hospitalarias están destinadas a contribuir el logro de la eficiencia y eficacia en la prestación de los servicios de enfermería; con la finalidad de mejorar la calidad de los cuidados ofrecidos al paciente, obviando actividades educativas que fortalezcan los comportamientos saludables del personal de enfermería.

Con respecto a los conocimientos sobre la realización de ejercicios físicos, se obtuvo un $49 \%$ en nivel de conocimiento medio (Tabla 2). Al respecto, López E. encontró un conocimiento acertado en un 52,4\%, en que el déficit de actividad física influye en el riesgo de enfermedades cardiovasculares (26). A pesar de que la mayoría tiene un conocimiento medio sobre los beneficios de la actividad física, las personas no la realizan; esto podría deberse a que asumen numerosas responsabilidades laborales y familiares que dejan poco espacio para la realización de ejercicio físico y el poco tiempo que les queda libre prefieren utilizarlo en otro tipo de ocio más sedentario.

En la dimensión conocimientos sobre hábitos alimentarios, tienen conocimiento alto solo un $8 \%$ (Tabla 2) a diferencia de López, que en su estudio encontró un conocimiento alto (66,7\%) (26). Los profesionales de la salud durante su formación académica reciben los conocimientos básicos acerca de una alimentación equilibrada. Por lo tanto, son personas privilegiadas en recursos cognitivos y prácticos para evitar conductas alimentarias que afecten su salud; sin 
embargo, los hábitos y costumbres puede estar fuertemente arraigados, lo que ocasiona una resistencia al cambio de conductas (6).

Con respecto al nivel de conducta de riesgo cardiovascular modificable referida por las enfermeras (Tabla 3), se obtuvo que un $45 \%$ tiene una conducta de alto riesgo. En la dimensión consumo de drogas legales referidas por las enfermeras con respecto al alcohol se encontró que un 14\% tiene una conducta de mediano riesgo con tendencia a alto riesgo (Tabla 4). Resultados similares reportó Hewit en el estudio estilos de vida en profesionales de la salud del Municipio de Zipaquirá donde un 46,57\% del personal de salud consume al menos dos tragos de alcohol al día, el cual es un porcentaje significativo (27). Asimismo, Vásquez et al., en el estudio factores de riesgo modificables para enfermedad cardiovascular en alumnos del programa de especialización en enfermería encontró que un $63 \%$ consume alcohol (28). Esto puede deberse porque desde temprana edad en cualquier reunión social las bebidas alcohólicas están presentes, debido a su aceptación en la realidad sociocultural y al fenómeno de la globalización, que ha influido en los patrones de consumo. Especialmente después de la Segunda Guerra Mundial, donde el consumo de alcohol dejó de estar asociado con las comidas, y se creó una nueva pauta de consumo, caracterizado por la ingesta de grandes cantidades en breve espacio de tiempo, asociada con actividades de ocio (29).

Con relación al consumo de drogas legales referido por las enfermeras, en el uso de tabaco se encontró que un $5 \%$ tiene conducta de alto riesgo con tendencia a mediano riesgo (Tabla 4). Sifuentes et al. encontraron que un $27 \%$ de la población posee hábitos tabáquicos (30). Asimismo Boggio en el estudio de alcohol y tabaco en enfermeras en un hospital público encontró que un $16 \%$ de enfermeras consume cigarrillos (13). A diferencia de Pérez et al., en el estudio de tabaquismo en las enfermeras de un Hospital Nacional de Lima, Perú; en el personal de enfermería de los servicios del Hospital Nacional Arzobispo Loayza encontró que el consumo actual de tabaco es solo de 3\% (31).

Asimismo, Vásquez et al.en un estudio sobre factores de riesgo modificables para enfermedad cardiovascular en alumnos del programa de especialización en enfermería encontró que un $8 \%$ es fumador (28). A pesar de que las políticas públicas exigen que se coloque en las cajetillas de cigarrillos la advertencia sanitaria que "fumar es dañino para la salud" existen profesionales de enfermería que lo consumen, incrementando el riesgo de padecer enfermedades cardiovasculares. El tabaco contiene un alcaloide nocivo llamado nicotina (32), que desencadena la liberación de las hormonas adrenalina, noradrenalina, y que producen daño en la pared de las arterias (33).

Cada vez hay más conciencia de la importancia del ejercicio físico y del deporte para la salud y la calidad de vida de las personas; sin embargo, en el presente estudio se observa que en cuanto a la dimensión ejercicio físico referido por las enfermeras un $87 \%$ tiene una conducta de alto riesgo (Tabla 4). Esto es similar al estudio de Díaz et al. sobre factores de riesgo para enfermedad cardiovascular en trabajadores de una institución prestadora de servicios de salud, donde la prevalencia de factores de riesgo cardiovascular estaba en la inactividad física 56,3\% (34).

La falta de ejercicio físico es un factor de riesgo que incrementa el peligro de padecer enfermedades cardiovasculares, diabetes y la obesidad (35). La falta de actividad física se evidencia cada vez a edades más tempranas por la larga permanencia frente al televisor, la computadora y los videojuegos en vez de realizar actividades deportivas, paseos y otras actividades al aire libre (36). Es probable que el desarrollo tecnológico este influyendo en los hábitos de las personas. Si bien los avances de la tecnología han contribuido a una mejora de los niveles de vida; la incorporación de nuevos conceptos de confort y bienestar han modificado el estilo de vida de los individuos, y no necesariamente mejorado su calidad de vida a largo plazo.

Con relación a los conocimientos sobre hábitos alimenticios referido por las enfermeras, se encontró una conducta de alto riesgo en un $86 \%$ debido a que no practican una dieta balanceada (Tabla 4). Similar a lo obtenido por Umaña en el estudio sobre relación entre estilos de vida y condición de salud de profesionales en enfermería del Hospital de Guápiles donde se encontró que un 39,53\% de las enfermeras no cuenta con un horario regular de comidas, mientras que un $41,86 \%$ está insatisfecho con el tiempo que el trabajo le otorga para alimentarse (37).

Asimismo, Aguilar et al., en el estudio relación entre los hábitos alimentarios y las manifestaciones digestivas más frecuentes en los estudiantes de posgrado en enfermería, un $31 \%$ tiene hábitos alimentarios malos. Respecto al consumo de comida rápida, condimentos y cremas: el $39 \%$ consume una vez por semana, un $38 \%$ de 2 a 4 veces por semana. Con respecto a las bebidas el $19 \%$ consume gaseosas (38). Hewit en el estudio estilos de vida en profesionales de la salud del Municipio de Zipaquirá encontró que un $40 \%$ consumen alimentos especiales o favoritos (grasa, chocolates, etc.), y le agregan sal a las 
comidas (27).

Es probable que estos resultados se asocien con una conducta alimentaria que está estructurada por múltiples variables que explican por qué una persona adopta, o no, un hábito alimentario en particular, como por ejemplo: la alimentación que se ofrece desde temprana edad que está enmarcada más en costumbres populares, que consideran ciertos alimentos beneficiosos para el desarrollo del niño; por tal razón, los conocimientos adquiridos en el aprendizaje de la carrera no tienen mucha influencia para el cambio de hábitos, cuando las personas llegan a su madurez (39). En el caso de las enfermeras, podría deberse que los trabajos de turno rotativo hacen que acudan a cafetines y restaurantes improvisados; donde los preparados están muy alejados de los criterios de una alimentación sana, y que esta aporte una cantidad de calorías acorde con el gasto energético de las personas.

Respecto a la relación entre nivel de conocimiento sobre factores de riesgo cardiovascular y nivel de conducta de riesgo cardiovascular modificable referido por las enfermeras (Tabla 5) se encontró que no existe relación significativa entre conocimiento y conducta de riesgo cardiovascular en las enfermeras, es decir; a conocimiento alto es poco probable que tenga conducta de riesgo bajo, $\mathrm{y}$ a bajo conocimiento es poco probable que haya conducta de riesgo alto; según prueba estadística chi cuadrado $(\mathrm{p}>0,05)$.

Los conocimientos deberían determinar la adopción de prácticas saludables porque permitan a las personas tomar decisiones informadas y optar por prácticas saludables o de riesgo frente al cuidado de su salud. Sin embargo, no dependen exclusivamente de la educación y la información, lo que puede significar que hay dificultad para la adopción de nuevas prácticas; como resultado de que los comportamientos están arraigados en las creencias y las tradiciones.

Los resultados obtenidos no concuerdan con lo estipulado en la hipótesis, contrariamente a lo esperado, los datos confirman que el personal de enfermería, a pesar de tener conocimiento medio en su mayor porcentaje, el comportamiento no es adecuado en la práctica de hábitos alimentarios, ejercicio físico en consumo de drogas legales como el alcohol y tabaco.

\section{REFERENCIAS BIBLIOGRÁFICAS}

1. World Health Organization. Word Health statistics 2012. Geneva, Switzerland: World Health Organization; 2012.

2. World Health Organization. Global Status report on
Noncommunicable Diseases 2010.Geneva, Switzerland: World Health Organization; 2011.

3. Reyes RM, Heredia LJ, Campodónico HS, Drago SJ, Alvarado CO. Registro nacional de infarto miocardico agudo. Revista Peruana de Cardiología. 2008; 34 (2): 85-99.

4. Fabregate M, Sabán RJ, Sánchez- Largo E, Coca D. Introducción al riesgo cardiovascular. Estudio Framingham. En: Sabán RJ. Control global del riesgo cardiometabólico. Madrid: Díaz de los Santos; 2012. p. 849-862.

5. Fernández E, Sabán J, Fabregate M, Fabregate R. Epidemiologia de la enfermedad cardiovascular. En: Sabán RJ. Control global del riesgo cardiometabólico. Madrid: Díaz de los Santos; 2012. p. 31-78.

6. Tenahua QI, Landeros OE, Linares FG, Grajales AI. Capacidades y acciones de autocuidado en personas con factores de riesgo cardiovascular. Enfermería en Cardiología. 2007; 14 (41):19-24.

7. Bonow RO, Mann DL, Zipes DP, Braunwald. Cardiología en atención primaria: prevención y poblaciones especiales. 9. ${ }^{\circ}$ ed. Madrid, España: Elsevier; 2012.

8. Organización Panamericana de la Salud. Alcohol y Salud Pública en las Américas. Un caso para la acción. Washington; 2007.

9. Organización Panamericana de la Salud. Informe sobre control del tabaco para la región de las Américas. Washington: Organización Panamericana de la Salud; 2011

10. Organización Panamericana de la Salud, organización mundial de la salud. Salud en las Américas. Panorama regional y perfiles de país. Volumen de países. Perú. Washington: Organización Panamericana de la Salud; 2012.

11. World Health Organization. Marco Mundial de Vigilancia integral, con inclusión de indicadores y un conjunto de objetivos mundiales de aplicación voluntaria para prevenir y controlar las enfermedades no transmisibles. Documento de debate revisado de la OMS. World Health Organization; 25 de julio del 2012. Disponible en: http://www.who.int/ nmh/events/2012/Discussion_paper3_ES.pdf

12. Fiestas F. Reduciendo la carga de enfermedad generada por el consumo de alcohol en el Perú: Propuesta basada en la evidencia. Revista Peruana de medicina experimental y Salud Pública. 2012; 29(1):112-18.

13. Boggio JM. Consumo de alcohol y tabaco en las enfermeras en un hospital público. Cadiz, España: Rev Portales médicos.com. Octubre del 2010.

14. Organización Panamericana de la Salud. Alcohol y atención primaria de la salud. Informaciones clínicas básicas para la identificación y el manejo de riesgos y problemas. Washington: Organización Panamericana de la Salud; 2008.

15. World Health Organization. Addiction to Nicotine: Gender, Women, and the Tobacco Epidemic. Geneva, Switzerland: World Health Organization; 2010. p. 139-149.

16. Ministerio de salud. El Humo de tabaco afecta la salud de todos. Lima: Ministerio de Salud; 2010. ( Citado el 24 de julio 2010). Disponible en:

http://www.minsa.gob.pe/portada/Especiales/2010/tabaco/ default.asp

17. World Health Organization. Impact of Tobacco Use on 
Women's Health: Gender, Women, and the Tobacco Epidemic. Geneva, Switzerland: World Health Organization; 2010. p. 51-62.

18. Sauvage LR. Prevenir las enfermedades del corazón: Diagnóstico, prevención y tratamiento. Madrid, España: Amat; 2011.

19. Márquez RS, Garatachea VN, Actividad física y Salud. Madrid: Díaz de los Santos; 2013.

20. Patiño FA, Márquez J (Coordinadores). Actividad física y ejercicio físico en salud: Retos en un contexto globalizado. Medellín: Funámbulos editores; 2009.

21. Cabrera A, Rodríguez PM, Rodríguez BL, et al. Sedentarismo: tiempo de ocio activo frente a porcentaje de gasto energético. Revista española de cardiología. 2007; 60(3):244-250.

22. Weinberg RS, Gould D. Fundamentos de Psicología del deporte y del ejercicio físico. 4ta ed. Madrid: Editorial médica panamericana S.A; 2010.

23. O’Donnell CJ, Elosua R. Factores de riesgo cardiovascular: perspectivas derivadas del FraminghamHeartStudy. Revista Española de Cardiología. 2008; 61(3): 299-310.

24. Winterkorn DL, Oliveira CM. La espiritualidad en el cuidado de sí para profesionales de enfermería. Rev. Latinoam Enfermagen. 2008; 16 (2).

25. Marriner TA, Raile AM. Modelos y Teorías de enfermería. Séptima edición. Madrid, España: Elsevier Mosby; 2011.

26. Lopez E. Conocimiento de los Factores de riesgo cardiovascular y comportamiento para la prevención del personal de enfermería. Venezuela: Universidad Centro Occidental Lissandro Alvarado; 2006.

27. Hewitt RN. Estilos de vida en profesionales de la salud del municipio de Zipaquirá. Psychología: Avances en la disciplina. 2007; 1(1): 61-97.

28. Vásquez VV, Olarte ChD, López MR, Alcántara SP. Factores de riesgo modificables para enfermedad cardiovascular en alumnos del programa de especialización en enfermería. Tesis de Grado.Lima, Perú: Universidad Peruana Cayetano Heredia; 2012.

29. CEDRO. El problema de las Drogas en el Perú. 25 años en la lucha contra las drogas y la mejora de la calidad de vida. Lima, Perú: CEDRO,; 2011.

30. Sifuentes. CA, Sosa GE, Pérez MA, Parra FF. Riesgo cardiovascular del personal de enfermería. Rev. Enfermería Global. 2011; 10(21): 1-10.

31. Pérez SV, Ferreira PS, Pillon SC. Tabaquismo en las enfermeras de un hospital nacional de Lima, Perú. Rev. Latino-Am. Enfermagem. 2010;18:550-556.

32. World Health Organization. Who report on the global tobacco epidemic, 2011 Warning about the dangers of tobacco. Geneva, Switzerland: World Health Organization; 2011.

33. Wilmore JH, Costill DL. Fisiología del esfuerzo y el deporte. 6. ${ }^{\circ}$ ed. Madrid, España: Paidotribo; 2007.

34. Díaz RJ, Muñoz MJ, Sierra TC. Factores de riesgo para enfermedad cardiovascular en trabajadores de una institución prestadora de servicios de salud, Colombia. Revista de Salud Pública. 2007; 9 (1):64-75.
35. Das P, Horton R. Rethinking our approach to physical activity. Lancet. 2012;380(9838):189-90. doi: 10.1016/ S0140-6736(12)61024-1.

36. Silverthorn D. Fisiología Humana un enfoque integrado. $4^{\circ}$ ed. Buenos Aires: Editorial Médica Panamericana; 2009.

37. Umaña MA. Relación entre estilos de vida y condición de salud de las (los) profesionales en enfermería, Hospital de Guápiles. Enfermería en Costa Rica. 2007; 28 (1): 5-11.

38. Aguilar CC, Alvarado GE, Rivera DJ, Relación entre los hábitos alimentarios y las manifestaciones digestivas más frecuentes en los estudiantes de post grado en enfermería. Tesis de Grado. Lima, Perú: Universidad Peruana Cayetano Heredia; 2011.

39. Rodríguez RV, Simón ME. Bases de la alimentación humana. Madrid, España: Gesbiblo; 2008.

\section{Correspondencia:}

Torres-Deza Clara

Correo electrónico: clara.torres@upch.pe

Fecha de recibido: 29 de noviembre de 2014

Fecha de aceptación: 26 de marzo de2015 